\title{
Bornes inférieures pour les marches aléatoires sur les groupes $p$-adiques moyennables
}

\author{
Sami Mustapha \\ Institut mathématique de Jussieu, 175, rue du Chevaleret, 75013, Paris, France \\ Reçu le 7 décembre 2004 ; accepté le 8 février 2005 \\ Disponible sur Internet le 22 juin 2005
}

Résumé

On donne des estimations centrales inférieures du noyau de transition d'une marche aléatoire symétrique sur un groupe $p$-adique moyennable.

(C) 2005 Elsevier SAS. Tous droits réservés.

\begin{abstract}
We give central lower estimates for the transition kernels corresponding to symmetric random walks on amenable $p$-adic groups.

(c) 2005 Elsevier SAS. Tous droits réservés.

MSC: 22E35; 60B15; 60G50

Keywords: Random walks; $p$-adic groups

\section{Introduction}

Soit $G$ un groupe localement compact et moyennable. Notons $\mathrm{d} g=\mathrm{d}^{l} g$ et $\mathrm{d}^{r} g$ respectivement les mesures de Haar gauche et droite sur $G$. Notons $m(g)=\mathrm{d}^{r} g / \mathrm{d}^{l} g$ la fonction module sur $G$ normalisée par $m(e)=1, e$ désignant l'élément identité. Soit $\mathrm{d} \mu(g)=\varphi(g) \mathrm{d}^{r} g \in \mathbf{P}(G)$ une mesure de probabilité sur $G$ où $\varphi(g)$ est supposée à support compact ou à décroissance rapide à l'infini. Supposons $\mu$ symétrique (i.e. stabilisée par l'involution $\left.g \rightarrow g^{-1}\right)$ et considérons la marche aléatoire sur $G$ induite par $\mu$, i.e. le $G$-processus $\left\{X_{n}\right\}_{n \geqslant 0}$ évoluant de la manière suivante : si $X_{n}=g$ à l'instant $n$ alors $X_{n+1}=g h, h$ étant choisi selon $\mu$. Notons $\mathrm{d} \mu^{* n}(g)=\varphi_{n}(g) \mathrm{d}^{r} g$ $(n=1,2, \ldots)$ les puissances de convolution successives de la mesure $\mu$. Une question centrale est de décider de la rapidité de la convergence de $\varphi_{n}(e) \rightarrow 0$ quand $n \rightarrow \infty$.
\end{abstract}

Adresse e-mail : sam@math.jussieu.fr (S. Mustapha). 
Dans le cas des groupes de Lie unimodulaires (et moyennables) la réponse à cette question est contenue dans le type de croissance du volume du goupe $G$. Rappelons que si $G$ désigne un groupe localement compact à génération compacte, la croissance du volume est définie de la manière suivante. On fixe $\Omega$ un voisinage compact, symétrique, de l'élément identité de $G$ tel que $G=\bigcup_{n \geqslant 0} \Omega^{n}$ où $\Omega^{n}=\Omega \cdot \Omega \cdots \Omega$ (n fois) et $\Omega^{0}=\{e\}$ et on pose (cf. [12])

$$
\gamma(n)=\operatorname{Vol}\left(\Omega^{n}\right), \quad n=1,2, \ldots
$$

où le volume est calculé relativement à la mesure de Haar (gauche ou droite) sur $G$. On pose

$$
|g|_{G}=\inf \left\{n, g \in \Omega^{n}\right\}, \quad g \in G .
$$

On définit une distance invariante à gauche sur $G$ en posant $d\left(g, g^{\prime}\right)=\left|g^{-1} g^{\prime}\right|_{G}$. La fonction $\gamma(n)$ apparaît alors comme la fonction donnant le volume de la boule centrée sur $e$ et de rayon $n$ dans $G$. Si $\Omega^{\prime}$ est un autre voisinage symétrique compact de $e$, engendrant $G$, alors les fonctions $\gamma(n), \gamma^{\prime}(n)$ qui correspondent respectivement à $\Omega$ et $\Omega^{\prime}$ vérifient l'équivalence $\gamma(n) \approx \gamma^{\prime}(n)$, i.e.

$$
\gamma^{\prime}(n) \leqslant C \gamma(C n)+C \leqslant C^{\prime} \gamma^{\prime}\left(C^{\prime} n\right)+C^{\prime}, \quad n \geqslant 1,
$$

où $C, C^{\prime}>0$ désignent des constantes convenables. Il en est aussi de même pour les distances associées à $\Omega$ et $\Omega^{\prime}$. C'est ce qui justifie la notation $|\cdot|_{G}$ (cf. $\left.[12,35]\right)$.

Pour les groupes de Lie on a la dichotomie suivante (cf. [12,16]) : soit

$$
\gamma(n) \approx n^{D}
$$

où $D=D(G)=1,2, \ldots$ est un entier qui ne dépend que du groupe $G$, soit

$$
\gamma(n) \approx e^{n} .
$$

Dans le premier cas on dit que $G$ est à croissance polynômiale (du volume) et dans le second on dit que $G$ est à croissance exponentielle. Pour les groupes de Lie moyennables unimodulaires le comportement de $\varphi_{n}(e)$ est lié à la croissance du volume de la manière suivante (cf. $[1,8,13,35])$ :

$$
\begin{aligned}
& \varphi_{n}(e) \approx n^{-D / 2} \Longleftrightarrow \gamma(n) \approx n^{D}, \\
& \varphi_{n}(e) \approx e^{-n^{1 / 3}} \Longleftrightarrow \gamma(n) \approx e^{n} .
\end{aligned}
$$

Pour les groupes de Lie moyennables non-unimodulaires le comportement de $\varphi_{n}(e)$ n'est plus décidé par la croissance du volume (ces groupes sont tous à croissance exponentielle) mais par la géométrie des racines de l'action du radical sur le nilradical (cf. [31,32]).

Le cas discret et plus généralement celui des groupes localement compacts à génération compacte est beaucoup plus délicat (cf. $[1,8,20-23,28-30,35,36])$. D'abord on ne dispose en général pas de dichotomie pour la croissance du volume pour les groupes moyennables (cf. [11]). D'autre part bien que l'on dispose dans le cas des groupes à croissance polynômiale de l'équivalence $\varphi_{n}(e) \approx n^{-D / 2}$ (où il faut se restreindre aux indices pairs pour l'estimation inférieure), on ne dispose, dans le cas des groupes unimodulaires à croissance exponentielle, que de l'estimation supérieure (cf. [14])

$$
\varphi_{n}(e) \leqslant C \exp \left(-c n^{1 / 3}\right), \quad n \geqslant 1
$$

En effet cette estimation supérieure n'est pas accompagnée, en général, d'une estimation inférieure analogue. Ch. Pittet et L. Saloff-Coste ont établi l'existence de groupes discrets résolubles, à croissance exponentielle pour lesquels la décroissance de $\varphi_{n}(e)$ est en $\exp \left(-c n^{\alpha}\right)$ avec $\alpha \in(0,1)$ pouvant être pris arbitrairement proche de 1 (cf. $[21,22])$. L'estimation inférieure $\varphi_{2 n}(e) \geqslant c \exp \left(-C n^{1 / 3}\right)$ est cependant vérifiée dans le cas des groupes polycycliques (cf. [1]). Pittet et Saloff-Coste ont récemment étendu ce résultat aux groupes résolubles finiment engendrés de rang de Prüfer fini (cf. [23]). Ils ont posé le problème de déterminer (cf. [23], §8) si une telle estimation inférieure était satisfaite dans le cas des groupes analytiques $p$-adiques. Nous donnons ici une réponse à ce problème (pour 
une réponse partielle dans le cas des produits semi-directs $\mathbf{Q}_{p}^{m} \bowtie\left(\mathbf{Q}_{p}^{*}\right)^{l}$ où il est possible de calculer explicitement $\left.\varphi_{n}(e) \mathrm{cf} .[17]\right)$.

Dans toute la suite on désigne par $k$ un corps commutatif, localement compact, non discret, totalement disconnecté, de caractéristique 0 et on considère $G$ un groupe algébrique connexe sur $k$. On suppose ce groupe moyennable et à génération compacte. On désigne par $\mathrm{d} \mu(g)=\varphi(g) \mathrm{d}^{r} g \in \mathbf{P}(G)$ une mesure de probabilité sur $G$, symétrique, de support compact. On note $\mathrm{d} \mu^{* n}(g)=\mathrm{d}(\mu * \cdots * \mu)(g)=\varphi_{n}(g) \mathrm{d}^{r} g$ les puissances de convolution successives de $\mu$. On suppose que la densité $\varphi$ est continue et qu'il existe $e \in \Omega=\Omega^{-1} \subset G$ tel que

$$
\inf \{\varphi(g), g \in \Omega\}>0, \quad G=\bigcup_{n \geqslant 0} \Omega^{n},
$$

où $e$ désigne l'élément identité de $G$.

Théorème 1. Avec les notations ci-dessus on a :

$$
\varphi_{2 n}(e) \geqslant c \exp \left(-C n^{1 / 3}\right), \quad n=1,2, \ldots,
$$

pour des constantes $c, C>0$ indépendantes de $n$.

Dans le cas où le groupe $G$ est unimodulaire, on a :

Théorème 2. Soient $G$ et $\mu \in \mathbf{P}(G)$ comme ci-dessus. Supposons le groupe $G$ unimodulaire, alors

$$
\frac{1}{C} \exp \left(-c_{1} n^{1 / 3}\right) \leqslant \varphi_{2 n}(e) \leqslant C \exp \left(-c_{2} n^{1 / 3}\right), \quad n=1,2, \ldots
$$

pour des constantes $c_{1}, c_{2}, C>0$ indépendantes de $n$.

Pour aider le lecteur à mieux situer les résultats ci-dessus rappelons que Varopoulos a montré dans [32] qu'une estimation inférieure en $\exp \left(-c t^{1 / 3}\right)$ était toujours satisfaite par le noyau de la chaleur associé à un sous-Laplacien (ou le noyau de transition d'une marche aléatoire) dans le cas où $G$ est un groupe de Lie réel connexe moyennable, unimodulaire ou non. Le Théorème 1 ci-dessus montre que ceci est aussi le cas pour les groupes analytiques $p$ adiques. Le cas $p$-adique présente cependant la particularité suivante. Si on suppose le radical unipotent de $G$ non trivial alors le fait d'être à génération compacte force le groupe $G$ à être à croissance exponentielle (cf. [24]). Si on suppose en plus que $G$ est unimodulaire on peut alors utiliser les résultats généraux de [14] qui entraînent l'estimation supérieure $\varphi_{n}(e) \leqslant C \exp \left(-c n^{1 / 3}\right), n=1,2, \ldots$. Cette estimation combinée avec l'estimation (1) entraîne (2). D'où le Théorème 2.

Les estimations inférieures dans le cas de la croissance polynômiale sont établies à partir d'estimations hors diagonales de type gaussien intégrées sur des boules de rayon convenable (cf. [14]). De telles estimations gaussiennes peuvent être établies dans le cas de la croissance exponentielle (cf. [10,18,34]) mais sont en générales insuffisantes pour permettre l'obtention d'estimations centrales inférieures (cf. néanmoins $[18,33]$ ).

On peut distinguer deux autres approches pour obtenir des estimations centrales inférieures : une approche géométrique (cf. [6,8,9]), qui consiste à construire des ensembles de Følner (cf. [19]) adaptés à des inégalités de type anti-Faber-Krahn (cf. [7]) qui permettent de déduire les bonnes estimations inférieures pour $\varphi_{n}(e)$ et une approche probabiliste (cf. [1,32]) qui consiste à établir des estimations inférieures pour les probabilités d'occurrence de certains évènements qui permettent d'estimer inférieurement $\varphi_{n}(e)$. L'approche géométrique dont le spectre d'application est assez large (variétés Riemanniennes, graphes, groupes de Lie, groupes discrets,...) semble utiliser d'une manière cruciale des propriétés de symétrie du noyau $\varphi_{n}(\cdot)$, ce qui, par exemple dans le cas des groupes de Lie, requiert l'unimodularité. Comme ici nous ne faisons pas une telle hypothèse nous utilisons la deuxième approche. 


\section{Racines de l'action adjointe}

Soit $G$ un groupe algébrique connexe sur $k$, un corps commutatif, localement compact, non discret, totalement disconnecté, de caractéristique 0 . Soient $Q$ et $U$ resp. le radical et le radical unipotent de $G$ (cf. [5,27]). On supposera $G$ moyennable, ce qui signifie que le groupe semi-simple $G / Q$ est compact (cf. [25]).

On sait que le radical $Q$ se décompose comme produit semi-direct $Q=U \bowtie A$, où $A$ est un groupe algébrique abélien constitué d'éléments semi-simples de $G$. Le groupe $A$ s'écrit aussi comme produit direct d'un tore $T$ et d'un groupe fini $F_{0}$ (cf. [5]).

Nous allons définir les racines de l'action de $T$ sur $U$ en nous appuyant sur la structure des corps locaux totalement disconnectés. Soit $|\cdot|$ la valeur absolue standard sur $k$ (cf. [2,4]). On pose $|k|=\{|x|, x \in k, x \neq 0\}$. On désigne par $p$ un générateur du groupe cyclique infini $|k|$ (on choisit $p=\left|x_{0}\right|$, où $x_{0}$ est une uniformisante de $k$ vérifiant $\left|x_{0}\right|>1$ ). Le groupe multiplicatif du corps $k$ est le produit direct du groupe des unités de $k$, i.e le groupe compact $\{x \in k,|x|=1\}$ et du groupe cyclique infini $\left\{x_{0}^{n}, n \in \mathbf{Z}\right\}$. Le tore $T$ étant isomorphe à $\operatorname{dim} T=d$ copies du groupe multiplicatif de $k$ (cf. [5]), il s'écrit $T \cong \mathbf{Z}^{d} \times K$, où $K$ est un sous-groupe compact de $T$. Notons

$$
\pi: T \rightarrow \mathbf{Z}^{d}
$$

fixons $\pi_{1}=\pi^{-1}\left(e_{1}\right), \ldots, \pi_{d}=\pi^{-1}\left(e_{d}\right) \in T, e_{1}, \ldots, e_{d}$ désignant la base canonique de $\mathbf{Z}^{d}$. Pour

$$
t=\pi_{1}^{n_{1}} \cdots \pi_{d}^{n_{d}} \tau
$$

où $n_{1}, \ldots, n_{d} \in \mathbf{Z}, \tau \in K$, on a

$$
\operatorname{Ad}(t)=\left(\operatorname{Ad} \pi_{1}\right)^{n_{1}} \cdots\left(A d \pi_{d}\right)^{n_{d}}(\operatorname{Ad} \tau) .
$$

Observons que du fait que la caractéristique de $k$ est nulle, il est possible d'identifier $U$ à son algèbre de Lie $\operatorname{Lie}(U)$ en utilisant l'application exponentielle (cf. [5,26]).

Soit $\bar{k}$ une extention finie de $k$ contenant toutes les racines $\left.\operatorname{de} \operatorname{det}\left(\operatorname{Ad}\left(\pi_{j}\right)-\lambda I\right)\right)=0, j=1, \ldots, d$. De la preuve du Lemme de Zassenhaus (cf. [15]) on déduit qu'il existe une décomposition de $\operatorname{Lie}(U) \otimes_{k} \bar{k}$ en une somme directe

$$
\operatorname{Lie}(U) \otimes_{k} \bar{k}=W_{1} \oplus \cdots \oplus W_{r}
$$

où les sous-espaces $W_{j}, 1 \leqslant j \leqslant r$, sont invariants par $A d\left(\pi_{i}\right), i=1, \ldots, d$, et telle que la restriction de chaque $A d\left(\pi_{i}\right)$ à $W_{j}$ soit la somme d'un scalaire $\lambda_{j}\left(\pi_{i}\right)$ et d'un endomorphisme nilpotent. Il existe alors une base de $\operatorname{Lie}(U) \otimes_{k} \bar{k}$ où chaque $A d\left(\pi_{i}\right)$ est représenté par une matrice diagonale par blocs dont chacun des blocs $B_{j}\left(\pi_{i}\right), j=1, \ldots, r$, est une matrice triangulaire supérieure avec des éléments diagonaux égaux à $\lambda_{j}\left(\pi_{i}\right)$. Pour $t=\pi_{1}^{n_{1}} \cdots \pi_{d}^{n_{d}} \tau \in T$ on pose $\tilde{t}=\pi_{1}^{n_{1}} \cdots \pi_{d}^{n_{d}}$ de sorte que $\operatorname{Ad}(t)=\operatorname{Ad}(\tilde{t}) \operatorname{Ad}(\tau)$. En posant

$$
\chi_{j}(t)=\chi_{j}(\tilde{t})=\lambda_{j}\left(\pi_{1}\right)^{n_{1}} \ldots \lambda_{j}\left(\pi_{d}\right)^{n_{d}}, \quad j=1, \ldots, r,
$$

on voit alors que $A d(\tilde{t})$ est représenté, dans la base de $\operatorname{Lie}(U) \otimes_{k} \bar{k}$ considérée ci-dessus, par une matrice diagonale par blocs dont chacun des blocs $B_{j}(\tilde{t}), j=1, \ldots, r$, est une matrice triangulaire supérieure avec des éléments diagonaux égaux à $\chi_{j}(t)$. Comme $\tau$ commute avec $\tilde{t}$ les espaces $W_{1}, W_{2}, \ldots, W_{r}$ sont stables par $A d(\tau)$. Nous appelons les homomorphismes $\chi_{j}: T \rightarrow \bar{k}^{*}$ définis par (7) racines de l'action de $T$ sur $U$.

Observons par ailleurs que la valeur absolue $|\cdot|$ possède une extension sur $\bar{k}$. Nous notons aussi cette extension $|\cdot|$. On a $|x| \in\left\{\bar{p}^{n}, n \in \mathbf{Z}\right\} \cup\{0\}$ pour $x \in \bar{k}$, où $\bar{p}$ est une puissance rationnelle de $p$ (cf. [4]). Afin d'alléger les notations nous continuons à noter $p$ le nombre $\bar{p}$.

Considérons pour $j=1, \ldots, r$ les valeurs absolues distinctes associées aux racines $\chi_{j}$, i.e. les homomorphismes définis de $T \rightarrow\left\{p^{n}, n \in \mathbf{Z}\right\}=p^{\mathbf{Z}}$, par $t \rightarrow\left|\chi_{j}(t)\right|$. Notons $\alpha_{1}, \alpha_{2}, \ldots, \alpha_{s}$ les différentes valeurs absolues associées aux racines $\chi_{j}$.

Il est facile de voir, en utilisant la caractérisation donnée dans [3], que le groupe $G$ est à génération compacte si et seulement si tous les $\alpha_{j}$ sont distincts de 1 (i.e. l'homomorphisme de $T \rightarrow p^{\mathbf{Z}}$ identiquement égal à 1). Remarquons que pour $t=\pi_{1}^{n_{1}} \cdots \pi_{d}^{n_{d}} \tau \in T$ :

$$
\alpha_{j}(t)=p^{\gamma_{j, 1} n_{1}+\gamma_{j, 2} n_{2}+\cdots+\gamma_{j, d} n_{d}}, \quad j=1, \ldots, s,
$$


où les $n_{j}=n_{j}(t) \in \mathbf{Z}$, où le $d$-uplet $\left(\gamma_{j, 1}, \cdots, \gamma_{j, d}\right) \in \mathbf{Z}^{d}$ ne dépend que de $\alpha_{j}$. Dans toute la suite on notera $L_{j}$, $j=1, \ldots, s$, la $\mathbf{Z}$-forme linéaire sur $\mathbf{Z}^{d}$ définie par (8), i.e.

$$
L_{j}\left(n_{1}, \ldots, n_{d}\right)=\gamma_{j, 1} n_{1}+\gamma_{j, 2} n_{2}+\cdots+\gamma_{j, d} n_{d}, \quad\left(n_{1}, \ldots, n_{d}\right) \in \mathbf{Z}^{d} .
$$

Les racines définies ci-dessus vont nous permettre d'analyser certains aspects géométriques de l'action de $T$ sur $\operatorname{Lie}(U)$.

\section{Un lemme ultramétrique et son interprétation géométrique}

Soit $u_{1}, u_{2}, \ldots, u_{\delta}$ une base fixée de $\operatorname{Lie}(U)$. Pour $x=x_{1} u_{1}+x_{2} u_{2}+\cdots+x_{\delta} u_{\delta} \in \operatorname{Lie}(U)$, on définit $\|x\|=$ $\max _{i}\left|x_{i}\right|$. $\|x\|$ vérifie l'inégalité ultramétrique $\|x+y\| \leqslant \max (\|x\|,\|y\|), x, y \in \operatorname{Lie}(U)$, et vérifie $\|\alpha x\|=|\alpha|\|x\|$, $\alpha \in k, x \in \operatorname{Lie}(U)$. On note $B=\{x \in \operatorname{Lie}(U),\|x\| \leqslant 1\}$. La boule unité ainsi définie est une partie compacte ouverte de $\operatorname{Lie}(U)$ vérifiant $: u+v \in B, \alpha u \in B$, pour $u, v \in B$ et $\alpha \in k$ tel que $|\alpha| \leqslant 1$. Les définitions cidessus s'étendent d'une manière évidente à $\operatorname{Lie}(U) \otimes_{k} \bar{k}$. Munissons $\operatorname{End}_{\bar{k}}\left(\operatorname{Lie}(U) \otimes_{k} \bar{k}\right)$ de la norme $\|A\|=$ $\sup _{\|u\| \leqslant 1}\|A u\|$. On a alors, pour $A, A^{\prime} \in \operatorname{End}_{\bar{k}}\left(\operatorname{Lie}(U) \otimes_{k} \bar{k}\right)$

$$
\left\|\left|A+A^{\prime} \|\right| \leqslant \max \left(\|A\| \mid\left\|A^{\prime}\right\|\right) .\right.
$$

Remarquons que pour $t=\pi_{1}^{n_{1}} \cdots \pi_{d}^{n_{d}} \tau \in T$ et $\operatorname{Ad}(t) \in \operatorname{End}_{\bar{k}}\left(\operatorname{Lie}(U) \otimes_{k} \bar{k}\right)$ on a (cf. (5))

$$
|||\operatorname{Ad}(t)||| \leqslant p^{C|t|_{T}}, \quad t \in T,
$$

où $|t|_{T}=\left|n_{1}\right|+\cdots+\left|n_{d}\right|$ et où $C>0$ désigne une constante positive.

Proposition 1. Les notations étant comme ci-dessus, soient $t_{1}, t_{2}, \ldots, t_{m} \in T$ et soient $u>0$ et $v>0$ tels que $\left|t_{j}\right|_{T} \leqslant u, j=1, \ldots, m$, et $\alpha_{k}\left(t_{1} \cdots t_{m}\right) \leqslant p^{v}, k=1, \ldots, s$. Alors il existe une constante $C>0$ telle que

$$
\left\|\operatorname{Ad}\left(t_{1}\right) \operatorname{Ad}\left(t_{2}\right) \cdots \operatorname{Ad}\left(t_{m}\right) \mid\right\| \leqslant p^{C u+C v} .
$$

La preuve de cette proposition repose sur le résultat élémentaire suivant :

Lemme 1. Soient $T_{j}=\left(t_{\alpha, \beta}^{j}\right) \in \mathcal{M}_{n \times n}(\bar{k})(j=1,2, \ldots, l) l$ matrices triangulaires supérieures strictes et $M_{j}=$ $\lambda_{j} I+T_{j}, j=1,2, \ldots, l$, où $\lambda_{j} \in \bar{k}^{*}$. Soient $N \in \mathbf{N}$ et $M \in \mathbf{N}$ tels que $\left\|\left.\left|T_{j} \| \leqslant p^{N},\right| \lambda_{j}\right|^{-1} \leqslant p^{N},\left|\lambda_{1} \cdots \lambda_{l}\right| \leqslant p^{M}\right.$. Alors

$$
\left\|M_{1} M_{2} \cdots M_{l}\right\| \leqslant p^{2 n N+M} .
$$

Preuve du Lemme 1. Il suffit d'observer que

$$
M_{1} M_{2} \cdots M_{l}=\lambda_{1} \lambda_{2} \cdots \lambda_{l} \sum_{\alpha, i_{j}}\left(\lambda_{i_{1}} \lambda_{i_{2}} \cdots \lambda_{i_{\alpha}}\right)^{-1} T_{i_{1}} T_{i_{2}} \cdots T_{i_{\alpha}}
$$

où tous les termes tels que $\alpha>n$ sont nuls et d'utiliser (10).

Preuve de la Proposition 1. On a (avec les notations de (6), (7))

$$
\left.\operatorname{Ad}\left(\tilde{t}_{j}\right)\right|_{W_{k}}=\chi_{k}\left(t_{j}\right) I+T_{j}, \quad j=1,2, \ldots, m, \quad k=1, \ldots, r,
$$

où $T_{j}$ est une matrice triangulaire supérieure stricte. D'autre part, pour $t_{j}=\pi_{1}^{n_{1}} \cdots \pi_{d}^{n_{d}} \tau \in T$

$$
\chi_{k}\left(t_{j}\right)=\chi_{k}\left(\pi_{1}^{n_{1}} \ldots \pi_{d}^{n_{d}}\right)=\chi_{k}\left(\pi_{1}\right)^{n_{1}} \ldots \chi_{k}\left(\pi_{d}\right)^{n_{d}} .
$$


Ainsi

$$
\left|\chi_{k}\left(t_{j}\right)\right| \leqslant p^{C\left|t_{j}\right|_{T}}, \quad\left|\chi_{k}\left(t_{j}\right)^{-1}\right| \leqslant p^{C\left|t_{j}\right|_{T}} .
$$

En combinant (11), (13) et (14) on déduit facilement que

$$
\left\|T_{j} \mid\right\| \leqslant p^{C\left|t_{j}\right|} \leqslant p^{C u} .
$$

Il suffit alors pour déduire (12) d'appliquer le Lemme 1 aux $\left.\operatorname{Ad}\left(\tilde{t}_{j}\right)\right|_{W_{k}}, k=1, \ldots, r, j=1,2, \ldots, m$.

\section{Preuve du Théorème 1}

Nous suivons de près [32]. Soit $G$ comme dans le Théorème 1. Le groupe $G$ (resp. $G / U$ ) se décompose comme produit semi-direct (resp. direct)

$$
\begin{aligned}
& G=Q \bowtie S=(U \bowtie A) \bowtie S \cong U \bowtie(A \times S), \\
& G / U \cong A \times S \cong T \times F_{0} \times S \cong \mathbf{Z}^{d} \times \tilde{K} \times S
\end{aligned}
$$

où $\tilde{K}$ et $S$ sont compacts; les notations sont celles de la Section 2. Ceci résulte de la moyennabilité du groupe $G$ et de la décomposition de Levi. Pour $z=t \sigma=\tilde{t} \tau \sigma \in A \times S \cong G / U \cong \mathbf{Z}^{d} \times \tilde{K} \times S$, on définit

$$
|z|_{G / U}=|\tilde{t}|_{T}=\left|n_{1}(\tilde{t})\right|+\cdots+\left|n_{d}(\tilde{t})\right|,
$$

où $\tilde{t}=\left(n_{1}(\tilde{t}), \ldots, n_{d}(\tilde{t})\right) \in \mathbf{Z}^{d}$. Soit $\mathrm{d} \mu(g)=\varphi(g) \mathrm{d}^{r} g \in P(G)$ et $\mathrm{d} \mu^{* n}(g)=\varphi_{n}(g) \mathrm{d}^{r} g$ comme dans le Théorème 1 . Soit $\xi_{1}, \xi_{2}, \ldots \in G$ une suite de variables aléatoires indépendantes équidistribuées de loi $\mathrm{d} \mu(g)$ et soit $X_{n}=\xi_{1} \xi_{2} \ldots, \xi_{n}, n=1,2, \ldots$, la marche aléatoire sur $G$ associée aux variables $\xi_{1}, \xi_{2}, \ldots,\left(X_{0}=e\right)$. On a, pour toute partie borélienne $A \subset G$ :

$$
\mathbf{P}_{e}\left[X_{n} \in A\right]=\int_{A} \varphi_{n}(g) \mathrm{d}^{r} g, \quad n=1,2, \ldots .
$$

La mesure $\mathrm{d} \mu(g)$ étant symétrique, on a

$$
\mathrm{d} \mu(g)=\mathrm{d} \mu\left(g^{-1}\right)=\varphi(g) \mathrm{d}^{r} g=\varphi\left(g^{-1}\right) \mathrm{d} g=\varphi(g) m(g) \mathrm{d} g,
$$

d'où l'identité

$$
\varphi\left(g^{-1}\right)=\varphi(g) m(g), \quad g \in G .
$$

On a aussi

$$
\varphi_{n}\left(g^{-1}\right)=\varphi_{n}(g) m(g), \quad g \in G, n=1,2, \ldots .
$$

D'autre part

$$
\varphi_{2 n}(e)=\int_{G} \varphi_{n}\left(g^{-1}\right) \varphi_{n}(g) \mathrm{d} g=\int_{G} \varphi_{n}(g) m(g) \varphi_{n}(g) \mathrm{d} g=\int_{G} \varphi_{n}(g)^{2} \mathrm{~d}^{r} g .
$$

L'inégalité de Schwarz appliquée à (15) donne alors

$$
\varphi_{2 n}(e) \geqslant \frac{\left(\mathbf{P}_{e}\left[X_{n} \in A\right]\right)^{2}}{|A|}, \quad A \subset G, n=1,2, \ldots
$$

où $|A|$ désigne la mesure de Haar droite de la partie $A$.

En utilisant le fait que $G$ se décompose comme produit semi-direct $G=U \bowtie(A \times S)$ on peut écrire

$$
X_{n}=\xi_{1} \xi_{2} \cdots \xi_{n}=u_{1} z_{1} u_{2} z_{2} \cdots u_{n} z_{n}, \quad n=1,2, \ldots
$$


où $\xi_{j}=u_{j} z_{j}$ avec $u_{j} \in U$ et $z_{j} \in A \times S, j=1,2, \ldots$ En utilisant les automorphismes intérieurs $x^{y}=y x y^{-1}$, $x, y \in G$, on peut réécrire

$$
\begin{aligned}
& X_{n}=u_{1} u_{2}^{z_{1}} u_{3}^{z_{1} z_{2}} \cdots u_{n}^{z_{1} \cdots z_{n-1}} z_{1} z_{2} \cdots z_{n}=\Gamma_{n} Z_{n}, \\
& \Gamma_{n} \in U, \quad Z_{n} \in A \times S, \quad n=1,2, \cdots .
\end{aligned}
$$

Pour $n=1,2, \ldots$ introduisons les évènements

$$
\begin{aligned}
& E_{n}=\left\{\left|Z_{n}\right|_{G / U} \leqslant n^{5 / 6}\right\}, \\
& E_{n}^{\prime}=\left\{\left|L_{j}\left(\zeta_{1}+\zeta_{2}+\cdots+\zeta_{k}\right)\right| \leqslant n^{1 / 3}, 1 \leqslant j \leqslant s, k=1, \ldots, n\right\}
\end{aligned}
$$

où $\zeta_{j}=\tilde{\pi}\left(z_{j}\right), j=1,2, \ldots ; \tilde{\pi}$ désignant la projection canonique $\tilde{\pi}: A \times S \rightarrow \mathbf{Z}^{d}$ et où les $L_{j}$ sont comme dans (9).

En utilisant la Proposition 1 et la formule de Campbell-Haussdorff on voit que

$$
\Gamma_{n}=\exp \left(\gamma_{n}\right), \quad \gamma_{n} \in \operatorname{Lie}(U), \quad\left\|\gamma_{n}\right\| \leqslant C p^{C n^{1 / 3}}, \quad n=1,2, \ldots
$$

où la constante $C>0$ est indépendante de $n$ et où les notations sont celles de la Section 3 .

On déduit de ce qui précède que l'évènement $E_{n} \cap E_{n}^{\prime} \subset\left[X_{n} \in A_{n}\right]$ où la partie $A_{n} \subset G=U \bowtie(A \times S)$ est définie par

$$
A_{n}=\exp \left(\left\{\|u\| \leqslant C p^{C n^{1 / 3}}\right\}\right)\left\{|z|_{G / U} \leqslant n^{5 / 6}\right\} .
$$

Il est facile de voir (par une désintégration de la mesure $\mathrm{d}^{r} g$ ) que

$$
\left|A_{n}\right| \leqslant C n^{5 d / 6} p^{C n^{1 / 3}}=\mathrm{O}\left(\exp \left(C n^{1 / 3}\right)\right) .
$$

Par ailleurs la probabilité $\mathbf{P}\left(E_{n}^{c}\right)$ peut être estimée inférieurement par

$$
\mathbf{P}\left(E_{n}^{c}\right) \geqslant \mathbf{P}\left[\left|\zeta_{1}+\zeta_{2}+\cdots+\zeta_{n}\right|_{\mathbf{Z}^{d}} \geqslant C n^{5 / 6}\right] \geqslant \exp \left(-C n^{2 / 3}\right)
$$

où $|\cdot|_{\mathbf{Z}^{d}}$ désigne la norme euclidienne dans $\mathbf{Z}^{d}$. L'estimation (18) résulte du fait que le noyau de transition de la marche aléatoire symétrique $\zeta_{1}+\zeta_{2}+\cdots+\zeta_{n} \in \mathbf{Z}^{d}$ vérifie des estimations gaussiennes inférieures (cf. par exemple [14]).

Enfin pour estimer $\mathbf{P}\left(E_{n}^{\prime}\right)$ il suffit d'utiliser l'estimation maximale inférieure (cf. [34], Appendix)

$$
\mathbf{P}\left[\left|\zeta_{1}+\zeta_{2}+\cdots+\zeta_{k}\right|_{\mathbf{Z}^{d}} \leqslant \alpha, 1 \leqslant k \leqslant n\right] \geqslant \frac{1}{C} \exp \left(-C \frac{n}{\alpha^{2}}\right), \quad n=1,2, \ldots, \alpha>0 .
$$

En choisissant $\alpha \approx n^{1 / 3}$ dans cette dernière estimation et en utilisant (18) on déduit que

$$
\mathbf{P}\left(E_{n} \cap E_{n}^{\prime}\right) \geqslant c e^{-C n^{1 / 3}}, \quad n=1,2, \ldots
$$

L'estimation (1) est une conséquence immédiate de (16), (17) et (19). Ceci complète la preuve du Théorème 1.

\section{Références}

[1] G. Alexopoulos, A lower estimate for central probabilities on polycyclic groups, Canad. J. Math. 44 (1992) 897-910.

[2] G. Bachman, Introduction to $p$-Adic Numbers and Valuation Theory, Academic Press, 1964.

[3] A. Borel, J. Tits, Groupes réductifs, Publ. Math. IHES 27 (1965) 55-150.

[4] J.W.S. Cassels, Local Fields, Cambridge University Press, 1986.

[5] C. Chevalley, Théorie des Groupes de Lie, tome III, Hermann, 1955.

[6] Th. Coulhon, Large time behaviour of heat kernels on Riemannian manifolds: fast and slow decays, in: Journées équations aux dérivées partielles, St-Jean-de-Monts, 1998, pp. 1-12.

[7] Th. Coulhon, A. Grigor'yan, On diagonal lower bounds for heat kernels on non-compact manifolds and Markov chains, Duke Math. J. 89 (1) (1997) 133-199. 
[8] Th. Coulhon, A. Grigor'yan, Ch. Pittet, A geometric approach to on-diagonal heat kernel lower bounds on groups, Ann. Inst. Fourier 51 (6) (2001) 1763-1827.

[9] A. Grigor'yan, Heat kernel upper bounds on a complete non-compact manifold, Rev. Mat. Iberoamericana 10 (2) (1994) $395-452$.

[10] A. Grigor'yan, Gaussian upper bounds for the heat kernel on arbitrary manifolds, J. Differential Geom. 45 (1) (1997) 33-52.

[11] R.I. Grigorchuk, Degrees of growth of finitely generated groups and the theory of invariant means, Izv. Akad. Nauk SSSR Ser. Mat. 49 (5) (1984) 939-985. English translation: Math. USSR-Izv. 25 (1985), 259-300.

[12] Y. Guivarc'h, Croissance polynomiale et périodes des fonctions harmoniques, Bull. Soc. Math. France 101 (1973) $333-379$.

[13] W. Hebisch, On heat kernels on Lie groups, Math. Z. 210 (1992) 593-605.

[14] W. Hebisch, L. Saloff-Coste, Gaussian estimates for Markov chains and random walks on groups, Ann. Probab. 21 (1993) 673-709.

[15] N. Jacobson, Lie Algebras, Interscience, 1962.

[16] J. Jenkins, Growth of connected locally compact groups, J. Funct. Anal. 12 (1973) 113-127.

[17] S. Mustapha, Random walks on unimodular $p$-adic groups, Preprint.

[18] S. Mustapha, Gaussian estimates for heat kernels on Lie groups, Math. Proc. Cambridge Philos. Soc. 128 (1) (2000) $45-64$.

[19] Ch. Pittet, Følner sequences on polycyclic groups, Rev. Mat. Iberoamericana 11 (3) (1995) 675-686.

[20] Ch. Pittet, L. Saloff-Coste, A survey on the relationship between volume growth, isoperimetry, and the behavior of simple random walk on Cayley graphs, with examples, Preprint, 1997.

[21] Ch. Pittet, L. Saloff-Coste, Amenable groups, isoperimetric profiles and random walks, in: Geometric Group Theory Down Under (Canberra, 1996), de Gruyter, Berlin, 1999, pp. 293-316.

[22] Ch. Pittet, L. Saloff-Coste, On random walks on wreath products, Ann. Probab. 30 (2) (2002) 1-30.

[23] Ch. Pittet, L. Saloff-Coste, Random walks on finite rank solvable groups, J. Eur. Math. Soc. 4 (2003) 313-342.

[24] C.R.E. Raja, On classes of $p$-adic Lie groups, New York J. Math. 5 (1999) 101-105.

[25] H. Rieter, Classical Harmonic Analysis and Locally Compact Groups, Oxford Math. Monograph, Oxford University Press, 1968.

[26] J.-P. Serre, Lie Algebras and Lie Groups, Benjamin, New York, 1965.

[27] T.A. Springer, Linear algebraic groups, in: A.N. Parshin, I.R. Shafarevich (Eds.), Algebraic Geometry IV, Springer-Verlag, 1993.

[28] N.Th. Varopoulos, Random walks on soluble groups, Bull. Sci. Math. 107 (1983) 337-344.

[29] N.Th. Varopoulos, A potential theoritic property of soluble groups, Bull. Sci. Math. 108 (1983) 263-273.

[30] N.Th. Varopoulos, Groups of superpolynomial growth, in: S. Igasi (Ed.), Harmonic Analysis (Sendai, 1990), ICM Satellite Conference Proceedings, Springer, 1991.

[31] N.Th. Varopoulos, Diffusion on Lie groups, Canad. J. Math. 46 (2) (1994) 438-448.

[32] N.Th. Varopoulos, Diffusion on Lie groups II, Canad. J. Math. 46 (5) (1994) 1073-1092.

[33] N.Th. Varopoulos, Hardy-Littlewood theory on unimodular groups, Ann. Inst. H. Poincaré Probab. Statist. 31 (4) (1995) $669-688$.

[34] N.Th. Varopoulos, The heat kernel on Lie groups, Rev. Mat. Iberoamericana 12 (1) (1996) 147-186.

[35] N.Th. Varopoulos, L. Saloff-Coste, Th. Coulhon, Analysis and Geomety on Groups, Cambridge Tracts in Math., vol. 102, Cambridge University Press, 1993.

[36] W. Woess, Random Walks on Infinite Graphs and Groups, Cambridge Tracts in Math., vol. 138, Cambridge University Press, 2000. 